\title{
MS41-03 | Nano-Crystallization: Applying the Methods of Macromolecular Crystallography for Small Molecules
}

Babor, Martin (University of Chemistry and Technology, Prague, Prague, CZE); Nievergelt, Philipp P. (University of Zurich, Zürich, $\mathrm{CH})$; Cejka, Jan (University of Chemistry and Technology, Prague, Prague, CZE); Spingler, Bernhard (University of Zurich, Zürich, $\mathrm{CH})$

We have recently developed a method for the crystallization of cationic moieties with the help of novel anion screen [2]. Salts that are at least water soluble at a concentration of $2 \mathrm{mg} / \mathrm{ml}$ water can be crystallized to directly yield single crystals. So far, we have shown that the new method works for organic cations by either vapour diffusion at the nanoliter scale (Nievergelt et al., 2018) or the under-oil technique [1]. In the presentation, we will show that we could successfully extend our method for the determination of protein and coordination complexes.

We thank the University of Zurich, the Czech Science Foundation (grant No. 16-10035S) and the Specific University Research (MSMT No. 21-SVV/2018) for financial support.

[1] Babor, M., Nievergelt, P. P., Čejka, J., Zvoníček, V. \& Spingler, B. (2019). IUCrJ 6, 145-151.

[2] Nievergelt, P. P., Babor, M., Čejka, J. \& Spingler, B. (2018). Chem. Sci. 9, 3716-3722. 